\title{
Reduction of Four-Wave Mixing in DWDM System using Electro-Optic Phase Modulator
}

\author{
Naif Alsowaidi ${ }^{1}$, Tawfig Eltaif ${ }^{2}$, Mohd Ridzuan Mokhtar ${ }^{3}$, Belal A. Hamida ${ }^{4}$ \\ ${ }^{1,2}$ Faculty of Engineering \& Technology, Multimedia University, Melaka, Malaysia \\ ${ }^{3}$ Faculty of Engineering, Multimedia University, Cyberjaya, Selangor, Malaysia \\ ${ }^{4}$ Department Electrical and Computer Engineering, International Islamic University Malaysia
}

\begin{tabular}{l} 
Article Info \\
\hline Article history: \\
Received Oct 24, 2017 \\
Revised Jan 4, 2018 \\
Accepted Jan 11, 2018 \\
\hline Keyword: \\
DSF \\
DWDM \\
EOPM \\
FWM \\
SMF \\
\hline
\end{tabular}

\begin{abstract}
In this paper, electro-optic phase modulator (EOPM) is used to reduce the effect of four-wave mixing (FWM), which is placed after 64 DWDMchannels multiplexer. It was found that the FWM is very sensitive to the phase deviation of the EOPM, and it can be reduced by introducing a phase shift between pulses. The simulation results confirmed the ability of the EOPM in improving the system performanceas indicated by the bit error rates. In term of comparison, the system of 64 channels based intensity modulated/ direct detection (IM/DD) transmission achieved bit error rate of $10^{-26}$ over $30 \mathrm{~km}$ and $70 \mathrm{~km}$ without and with EOPM, respectively.
\end{abstract}

Copyright (c) 2018 Institute of Advanced Engineering and Science. All rights reserved.

\section{Corresponding Author:}

Tawfig Eltaif,

Faculty of Engineering \& Technology,

Multimedia University,

75450; Bukit Beruang, Melaka, Malaysia.

Email: tefosat@ieee.org

\section{INTRODUCTION}

Optical fiber offers huge bandwidth utilized by dense wavelength division multiplexing (DWDM) network [1], [2]. Nonlinear effects have a huge impact on the performance of DWDM; mainly four-wave mixing (FWM) phenomena. The FWM mainly occurs due to the interaction between two or more wavelengths, in which new wavelengths are generated due to the variations in the refractive index of the fiber [2]. Thus, the newly generated wavelengths may coincide with the original wavelengths resulted in introducing server source of impairments.

The phase of the signal in On-Off keying modulation (OOK) is an extra degree of freedom since the data is carried by signal's intensity and the inter and intra-channel FWM consider as the major source of impairments in high data rate OOK system. Hence, by using alternate-phase return-to-zero (APRZ) modulation, the phase of an RZ signal is modulated at half the bit rate in order to give a phase shift to neighboring bits which causes the intra-channel FWM contributions to interfere destructively [3]. Although such approach has been shown to be able to reduce the intra-channel FWM effects, however, it has been tested only for a single channel. In [4]; asynchronous phase modulation (APM) proposed and resulted in increasing the power tolerance of NRZ-OOK over a long distance. Similarly, in [5] an optical phase conjugation (OPC) firstly proposed for suppression the FWM effect. Unfortunately, the OPC possess several limitations as it is very complex, possess extra loss, and it cost too much. Recent studies in [6-9] used different modulation formats in DWDM system to increase the nonlinear tolerance such as the return to zero (RZ), carrier suppression return to zero (CSRZ), Differential phase shift keying (DPSK), Differential Quadrature Phase Shift Keying (DQPSK), optical duo-binary (ODB) and duo-binary return-to-zero (DRZ). The results of these studies revealed that CSRZ and DRZ modulations have better performance and are 
suitable for long transmission distance. Whilst in [10] the nonlinear effects in WDM system were overcome by the use of Gain Flattening Filter (GFF) in conjunction with Fiber Bragg Gratings (FBG).

Moreover, dispersion management has the ability to suppress the nonlinearity effects [11]; however, the range of the available wavelengths is limited in order to apply for the compensation besides the complication management which is required in some cases. In 2016 Bhagwan et al proposed new all-optical signal regeneration for $10 \mathrm{Gbps}$ transmission system to mitigate the nonlinear impairment, where the system has been tested only for a single channel at the transmitted power of $0 \mathrm{dBm}$ [12]. On the other hand, the influence of FWM in a virtual carrier (VC)-assisted direct detection multi-band orthogonal frequency division multiplexing metro network implementing ultra-dense WDM was theoretically evaluated in [13]. It was found that the generated products of the VC-VC -band FWM limits the performance of the system when the channel count is high. Moreover, the optical bistability and FWM in a hybrid Opto-mechanical system has been theoretically investigated [14]; the authors found that the peak intensity value of FWM and the distance between two peaks in the spectrum of FWM can be controlled by the Rabi frequency and the qubitresonator coupling strength respectively. Furthermore, linear polarization technique has been used for suppression FWM in [15], this technique has drawbacks such as; it is only applicable for WDM system in which the number of channels is not many, additional loss is introduced, and not effective at high launch power.

Recently, a hybrid scheme combining suboptimum channel separation (S-CS) and equal channel separation (ECS) schemes was presented in order to reduce the effect of FWM and increase the spectral bandwidth [16]. In this hybrid scheme, an algorithm was designed for finding actual locations of the original frequency channels, which helps to optimize the trade-off between the spectral bandwidth requirements and immunity from the FWM effect.

In this article, a technique based on electro-optic phase modulator (EOPM) to suppress FWM effects in 64 WDM channels system with equal channel spacing, has been proposed. Where a single EOPM placed after the WDM multiplexer to introduce a phase shift $\emptyset_{\text {EOPM }}$ between pulses. the suppression of FWM is taken place because of the phase deviation which was introduced by EOPM. DWDM system is simulated at $10 \mathrm{Gbps}$ using two types of fibers, single mode fiber and dispersion shifted fiber. The effects of a number of wavelengths, channel spacing and channel length on the performance of the system are investigated.

\section{SYSTEM DESCRIPTION}

Electro-optic phase modulator (EOPM) is placed after the WDM multiplexer as shown in Figure 1. The EOPM is used to introduce a phase shift between pulses. Where, the phase shift is actually the responsible for reducing inter and intra-channel FWM effects. Mathematically, the transmitted signals with and without EOPM device can be represented in Equations (1) and (2), respectively.

$$
\begin{aligned}
& S(t)=\sum_{i=1}^{N} \sqrt{\frac{E_{b}}{T}} e^{j 2 \pi f_{i} t} \\
& S(t)_{E O P M}=\left[\sum_{i=1}^{N} \sqrt{\frac{E_{b}}{T}} e^{j 2 \pi f_{i} t}\right] e^{j \phi_{E O P M} \sin \left(2 \pi f_{R F} t\right)}
\end{aligned}
$$

where $S(t)$ is the transmitted signals, $E_{b}$ is the pulse energy per transmitted bit, $T$ is a bit period, and $f_{R F}$ is the RF signal frequency. The overall flow of the transmission system is as shown in Figure 1.

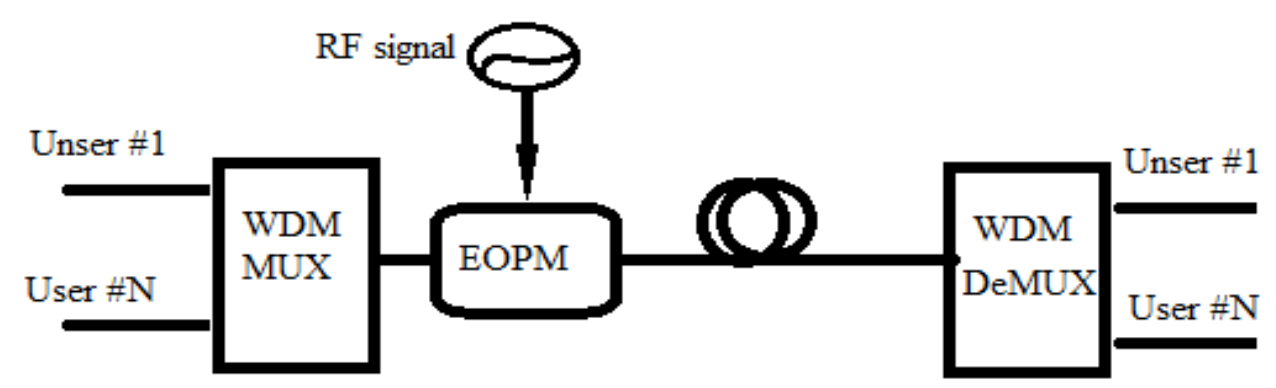

Figure 1. WDM optical transmission system with EOPM 
Two types of fibers namely, single mode fiber (SMF), and dispersion shifted fiber (DSF) used for 4 and 64 WDM channels system with an equal channel spacing (i.e., 50GHz) to confirm and ensure the effectiveness of EOPM with RF signal frequency $10 \mathrm{GHz}$ for improving the system performance by suppressing the FWM nonlinear effects. In addition, the system has been tested for different transmission distance. The typical system parameters are as summarized in Table 1 . The final results presented in this paper are based on intensity modulated/ direct detection (IM/DD) transmission using NRZ-OOK modulation scheme.

Table 1. System Parameters

\begin{tabular}{lr}
\hline \multicolumn{1}{c}{ Parameter } & \multicolumn{1}{c}{ Value } \\
\hline Number of channels & $\mathrm{N}=4 ; \mathrm{N}=64$ \\
Transmitted power & $14,26 \mathrm{dBm}$ \\
Channel spacing & $50 \mathrm{GHz}$ \\
Cross effective area for SMF, & $80 \mathrm{um}^{2}$ \\
Nonlinear refractive index & $2.6 \mathrm{e}-20 \mathrm{~m}^{2} / \mathrm{W}$ \\
Attenuation & $0.2 \mathrm{~dB} / \mathrm{k}$ \\
Dispersion for SMF & $17 \mathrm{ps} / \mathrm{nm}^{-k m}$ \\
Dispersion slope for SMF & $0.08 \mathrm{ps} / \mathrm{nm}^{2} \cdot \mathrm{km}$ \\
Cross effective area for DSF & $50 \mathrm{um}$ \\
Dispersion for DSF & $1 \mathrm{ps} / \mathrm{nm}^{2} \cdot \mathrm{km}$ \\
Dispersion slope for DSF & $0.075 \mathrm{ps} / \mathrm{nm}^{2} \cdot \mathrm{km}$ \\
Phase deviation & $\pi / 6$ \\
RF frequency & $10 \mathrm{GHz}$ \\
\hline
\end{tabular}

\section{RESULTS AND DISCUSSION}

The results demonstrate significant improvements in the overall system performance compare to that without EOPM as indicated by their bit error rates. The system improvements were found in both of the $4 \mathrm{WDM}$ and $64 \mathrm{WDM}$ channel systems. The performance of the 4 WDM channels system operating at $(1552.12 \mathrm{~nm}-1553.32 \mathrm{~nm})$ with a transmitted power $14 \mathrm{dBm}$ and carrying users' data at a data rate of $10 \mathrm{Gbps}$, is presented in Figure 2. The result clearly shows that the EOPM module has good performance in terms of the bit error rate $\left(10^{-24}\right)$ as compared with the one without EOPM $\left(10^{-18}\right)$ in all the transmitted power $(\mathrm{dBm})$.

Additionally, the proposed module of EOPM shows that increasing the channel spacing more than $100 \mathrm{GHz}$ doesn't make too much difference in terms of the bit error rate as shown in Figure 3.

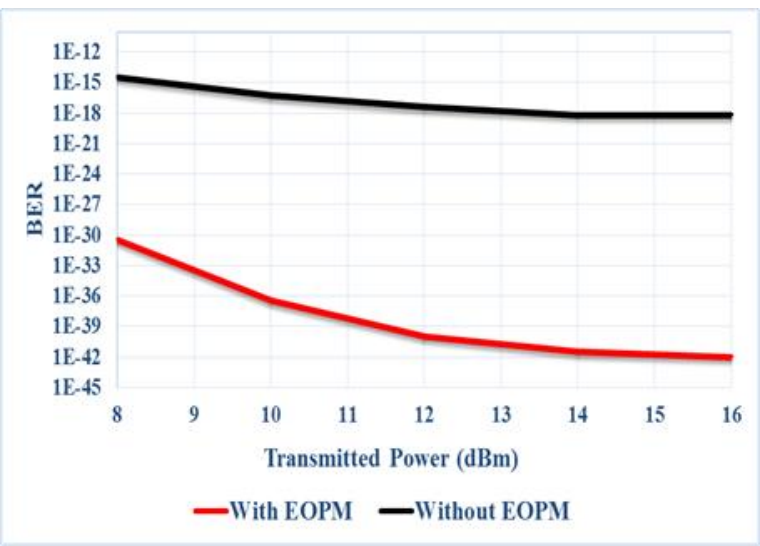

Figure 2. BER of 4-WDM channels with/without EOPM

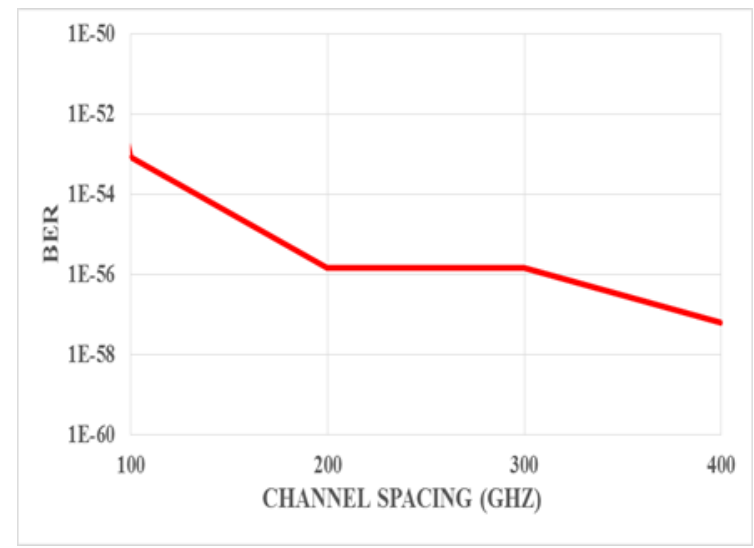

Figure 3. BER of 4-WDM channels with EOPM using different channel spacing

The Portions of the received signal in the time domain with and without EOPM are presented in Figure 4(a) and Figure 4(b), respectively. The shadow pulses (e.g. in,5.84ns-5.86ns window) in Figure 4(a) which are due to the intra-channel FWM effects clearly suppressed by EOPM as reveals in Figure 4(b). Comparing the pulses in Figure 4(b) to Figure 4(a), they were widened because of the EOPM employed. 


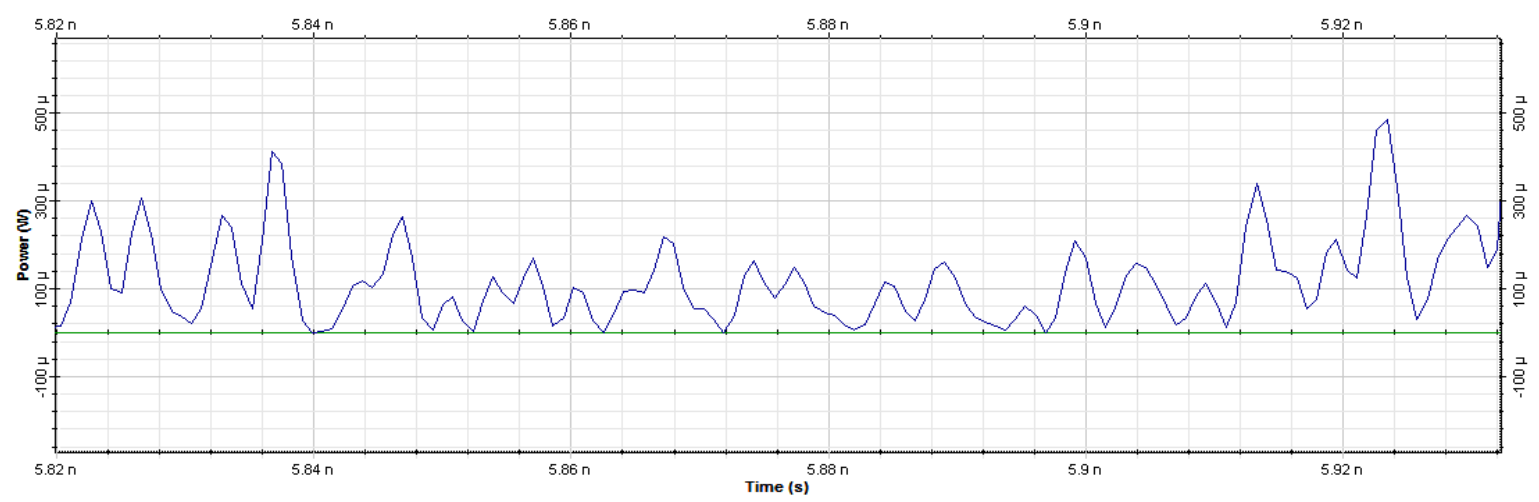

(a)

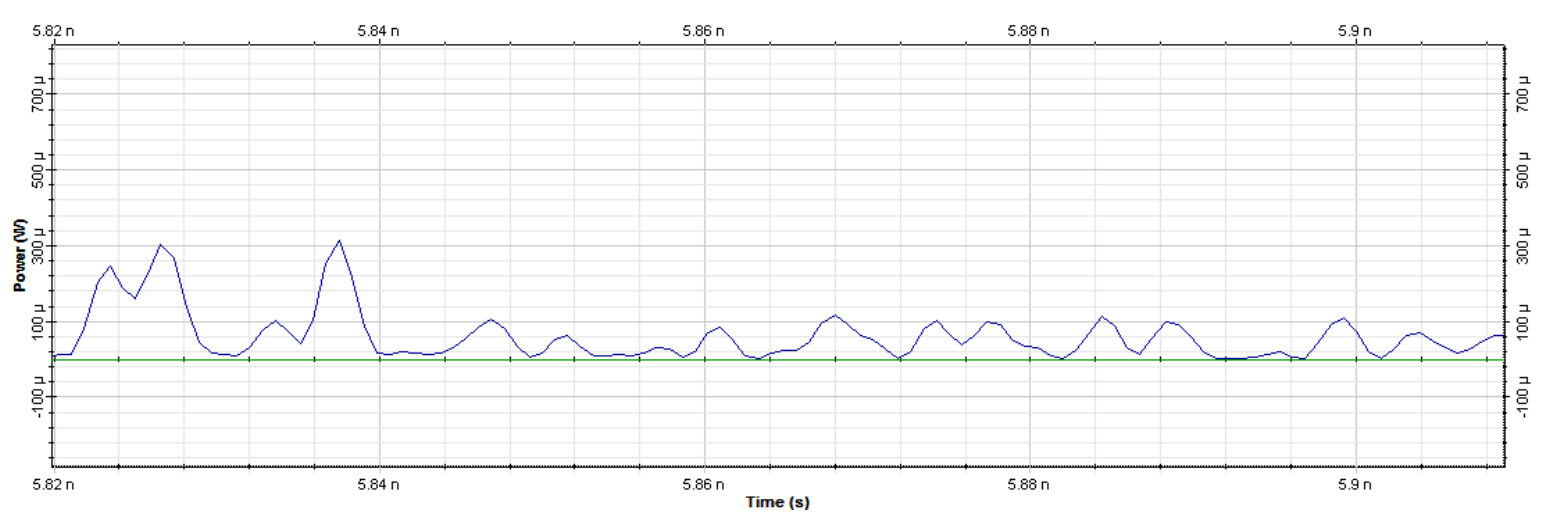

(b)

Figure 4. (a) Portions of the Received Signal in time domain without EOPM, (b) Portions of the Received Signalin time domain with EOPM

To further validate the improvements in the performance of the system, we simulated the EOPM module using SMF and DSF and compare their performance for selection purpose. The results found that the system with SMF has better performance as compared to the one with DSF when the transmission length is at $55 \mathrm{~km}$ or less as shown in Figure 5. However, as the transmission length increases, the system with DSF improves its performance, in which at $90 \mathrm{~km}$ the recorded BER of the system is $10^{-43}$ using DSF and $10^{-5}$ using SMF. Therefore, it is recommended to use the system with EOPM using the SMF if the distance is less than $55 \mathrm{~km}$ with specifications stated in Table 1.

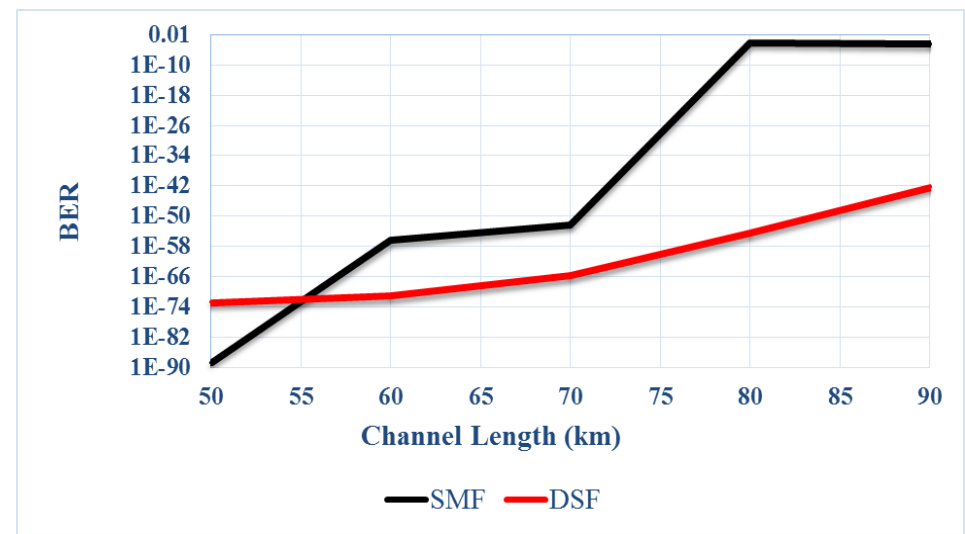

Figure 5. Comparison of 4-channel system with EOPM using SMF/DSF 
The FWM effect becomes very serious if the transmitted power is high or the number of channels is large, hence, the 64-WDM channels system with EOPM has been examined at a transmission power of 26 $\mathrm{dBm}$. Figure 6 shows the performance of the system with and without EOPM device. It was found that using EOPM with phase deviation, $\pi / 6$, and RF signal frequency of $10 \mathrm{GHz}$, the efficiency of FWM can be totally suppressed even at a high transmitted power and a large number of channels. As a comparison, EOPM proposal outperforms linear polarization for paring wavelengths (LPFPW) [15]. In addition, in Ref [16], the authors indicated that huge number of channels have bad performance in terms of BER due to the effect of FWM, while in our proposed system using EOPM, all the users show good performance, where the results presented in this paper is an average of all the users as shown in Figure 6.

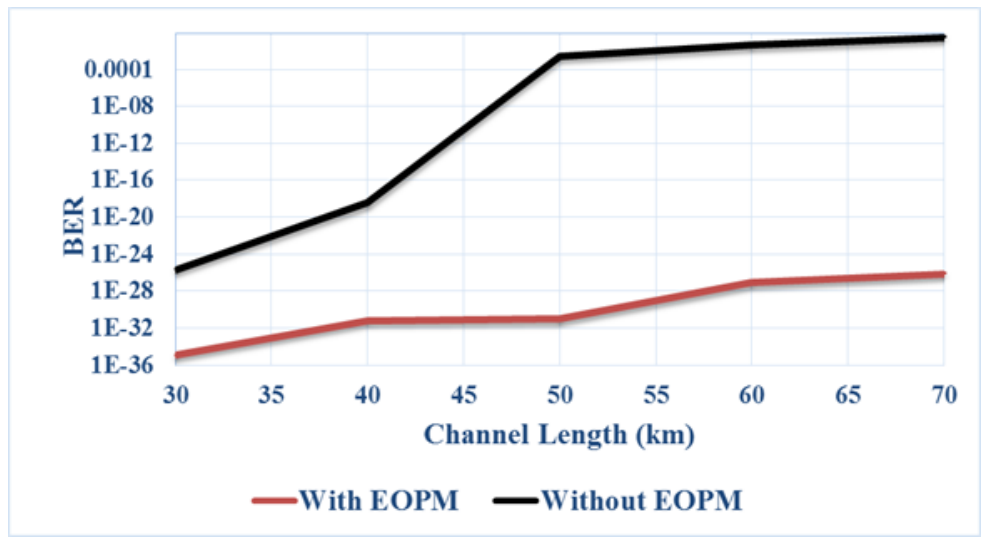

Figure 6. Comparison of 64-channel system with/out EOPM using SMF

\section{CONCLUSION}

An enhancement approach which based on electro-optic phase modulator (EOPM) for suppressing FWM effect in DWDM system has been implemented. FWM effect is very sensitive to the phase deviation of EOPM and RF signal frequency, and it was found that the FWM effect can be totally suppressed when the phase deviation set at the optimum value, $\pi / 6 \mathrm{rad}$ and the RF signal frequency set at $10 \mathrm{GHz}$. The results confirm the ability of the EOPM method in improving the system performance by reducing the efficiency of Inter and intra-channel FWM even at a high transmitted power and a large number of channels as it has been examined with 4 channels at $14 \mathrm{dBm}$ as transmitted power and 64 channels at $26 \mathrm{dBm}$. It was found that the 4 WDM channels system with EOPM using SMF has better performance if the channel length is less than $55 \mathrm{~km}$, however, if the channel length is greater than $55 \mathrm{~km}$, it is recommended to use DSF rather than SMF.

\section{ACKNOWLEDGEMENTS}

This work was supported by Multimedia University (Malaysia), project SAP ID: MMUI/160092.

\section{REFERENCES}

[1] Rana M, "Dense Wavelength Division Multiplexing Optical Network System", International Journal of Electrical and Computer Engineering (IJECE), 2012, vol. 2, no. 2, pp. 203-206.

[2] Agrawal G, "Nonlinear fiber optics", $5^{\text {th }}$ ed., Amsterdam: Academic Press, 2013, pp. 397-457.

[3] Forzati M, Berntson A, Martensson J, "Performance Analysis of Single-MZM APRZ Transmitters", J. Lightw. Technol., 2006, vol. 24, no. 5, pp. 2006-2014.

[4] Forzati M, Berntson A, Martensson J, "Asynchronous Phase Modulation for the Suppression of IFWM", J. Lightw. Technol., 2007, vol. 25, no. 10, pp. 2969-2975.

[5] Pechenkin V, FairIvan J, "On Four-Wave Mixing Suppression in Dispersion-Managed Fiber-Optic OFDM Systems With an Optical Phase Conjugation Module", J. Lightwave Tech., 2011, vol. 29, no. 11, pp. 1678-1691.

[6] LiL, Jian-yi W, Hong-anL, Xiu-taiZ, "Analysis Modulation Formats in DWDM Transmission System", TELKOMNIKA (Telecommunication Computing, Electronics and Control), 2013, vol. 11, no. 1, pp. 536-543.

[7] Xiancheng F, Xiaopeng L, Qinghua S. Research on OSNR and BER of 40G DWDM system on DRZ. TELKOMNIKA Indonesian Journal of Electrical Engineering. 2013; 11(2): 948-953. 
[8] Li L, Jian-yi W, Xiu-tai Z, Hong-an L, "Research on Mixed Data Rate and Format Transmission in WDM Networks", TELKOMNIKA (Telecommunication, Computing, Electronics and Control), 2013, vol. 11 no. 1, pp. 127-136.

[9] Li L, Yan-Tao C, Ji-Jun Z, Zhi-Rui L, Wan-Li C, "Research on the Modulation Performance in GPON System", TELKOMNIKA (Telecommunication Computing, Electronics and Control), 2014, vol. 12, no. 10, pp. 7304-7310.

[10] Mounia C, Otman A, Badiaa A, Fahd C, Ana Vazquez A, Mounir E, "Gain Flatness and Noise Figure Optimization of C-Band EDFA in 16-channels WDM System using FBG and GFF", International Journal of Electrical and Computer Engineering (IJECE), 2017, vol. 7, no. 1, pp. 289-298.

[11] Du J, Teng Z, Shen N, "Semi-analytic modeling of FWM noise in Dispersion-managed DWDM systems with DQPSK/DPSK/OOK channels", Opt. Commun., 2016, vol. 358, pp. 180-189.

[12] Das B, Abdullah M, Mohd Shah N, "A New All-Optical Signal Regeneration Technique for 10 GB/S DPSK Transmission System”, International Journal of Electrical and Computer Engineering (IJECE), 2016, vol. 6, no. 2, pp. 859-869.

[13] Cruz P, Alves T, Cartaxo A, "Theoretical Analysis of the Four-Wave Mixing Effect in Virtual Carrier-Assisted DD MB-OFDM Ultradense WDM Metropolitan Networks", J. Lightwave Tech., 2016, vol. 34, no. 23, pp. 5401-5411.

[14] Jiang L, Yuan X, Cui Y, Chen G, Zuo F, Jiang C, "Optical bistability and four-wave mixing in a hybrid optomechanical system", Physics Letters A., 2017, vol. 381, no. 38, pp. 3289-3294.

[15] Abd H, Din N, Al-Mansoori M, Abdullah F, Fadhil H, "Four-Wave Mixing Crosstalk Suppression Based on the Pairing Combinations of Differently Linear-Polarized Optical Signals", The Scientific World Journal, 2014, pp. 1-10.

[16] Singh G, Singh M, "Spectral bandwidth-efficient four-wave mixing minimization scheme for C-band dense wavelength division multiplexed system", Optical Engineering, 2017, vol. 56, no. 7, pp. 076-115.

\section{BIOGRAPHIES OF AUTHORS}

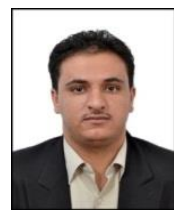

Naif Alsowaidi received the B.Eng. degree (with honors) in communication engineering from International Islamic University Malaysia (IIUM), in 2012 and the M.Sc. degree. in engineering science from Multimedia University (MMU), Malaysia, in 2015, currently he is a Ph.D. candidate at Multimedia University. His current research interests include optical communication, Optical CDMA systems, and nonlinear optics.

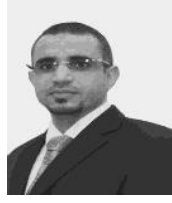

Tawfig Eltaif received his BSc degree in communication engineering from University of Tripoli, Libya, 2003, and MSc degree in microelectronics and Ph.D degree in micro-engineering \& nanoelectronics from National University of Malaysia, Malaysia, 2005, 2009, respectively. He joined Photronix Technologies Company, 2008-2011, as a product and research engineer. Since 2012 he has been a senior lecturer at Multimedia University. His current research is focused on optical communication, optical frequency comb, FBG, and optical amplifiers.

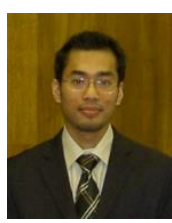

Mohd Ridzuan Mokhtar received his Ph.D degree in optoelectronics from the University of Southampton, UK, in 2005. He is professor at the Faculty of Engineering, Multimedia University, Malaysia. He became a research fellow with City University London, UK, for a year in 2009, sponsored by the Technology Strategy Board in the UK. He has been the director of research and collaboration at Multimedia University since 2011. His main research interests include fiber optic sensors and optical communications.

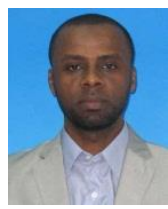

Belal Ahmed Hamidah received his BSc degree in Computer Engineering from Faculty of Engineering Tajoura, Libya, Sep 1996, MSc degree in microelectronics from University Kebangsaan Malaysia UKM, Malaysia, in July 2007 and Ph.D degree in Electrical and Computer EngineeringInternational Islamic University Malaysia, in Feb 2013. He was appointed as full-time postdoctoral researcher in Kulliyyah of Engineering-International Islamic University Malaysia (June- 2013-June2014). His research in the field of optical communication systems. Since 2014 he has been an assistant professor in International Islamic University Malaysia. His current research interests are Optical amplifier, Q-switched fiber lasers, Mode locked fiber laser, passive optical networks PONs and optical code division multiple access OCDMA. 